\section{Linear Array of Metallic Waveguides Focuses Surface Plasmon Polaritons}

One route to overcoming the diffraction limit in conventional optical devices is to utilize surface-plasmon polaritons (SPPs), which arise from the collective excitation of electrons at the interface between a conductor and an insulator. A standard method for generating surface plasmons is to shine light of the appropriate wavelength on a corrugated metal surface. An array of metallic waveguides not only can support but also can control the propagation of surface plasmons, as shown by X. Fan and G.P. Wang at Wuhan University, China, in the May 1 issue of Optics Letters (p. 1322). Through numerical simulations based on a finite-difference time-domain method, the researchers explain how a linear array of nanoscale waveguides made of silver can focus the propagating SPPs.

The plasmonic structure consists of an array of 30 identical waveguides arranged parallel to each other. Each waveguide is $2 \mu \mathrm{m}$ long and $20 \mathrm{~nm}$ wide, and the spacing between adjacent waveguides is $30 \mathrm{~nm}$. The waveguide metallization is a 20-nm-thick layer of Ag. There is a 20-nm air layer between the Ag layer and the substrate. Because of this design, the permittivity of the structure is periodically modulated, which can produce a negative refraction of the electromagnetic radiation. When the array is illuminated by a Gaussian optical beam focused at a point in front of the entrance facet of the array, the SPPs excited by the incident light are focused within the array. This occurs, according to the researchers, because the phase wavefront of the radiation entering the array is not planar but tilted with respect to the entrance facet. In contrast to the rays that enter the array in regions of dielectric only (which experience normal refraction), the rays penetrating the waveguide regions containing metallization change directions, effectively causing the light in those regions to experience negative refraction. In a similar fashion, the SPPs excited on each waveguide couple in an anomalous way and undergo negative refraction too, resulting in a focused spot of SPPs, said the researchers. In particular, report the researchers, a Gaussian optical beam with full width at half maximum of $265 \mathrm{~nm}$ positioned $250 \mathrm{~nm}$ in front of the waveguide can focus SPPs to a linewidth of $160 \mathrm{~nm}$. The researchers said that the focusing of the SPPs could potentially be augmented by using convex or concave structures at the entrance to the waveguide array as well as by using thinner metallization layers. The researchers said that these metallic waveguide arrays can find important applications in subwavelength optical systems-for example, as optical interconnects in advanced nanophotonic devices.

TUSHAR PRASAD

\section{Molecularly Imprinted Polyurethane Offers Economically Viable Route to Blood Typing}

Although blood typing according to $\mathrm{ABO}$ antigens is common, the process that is typically used requires expensive and difficultto-store antibodies. In the April 10 issue of
Angewandte Chemie International Edition (p. 2626; DOI: 10.1002/anie.200502857), O. Hayden, K.-J. Mann, S. Krassnig, and F.L. Dickert of the University of Vienna reported the use of molecularly imprinted polyurethane to identify the blood group of red blood cells (RBCs) both in suspension and in whole blood. In addition to introducing a potentially more economical method for typing blood than is currently available, their research demonstrates the use of molecular imprinting for analytes larger than those that have been previously used.

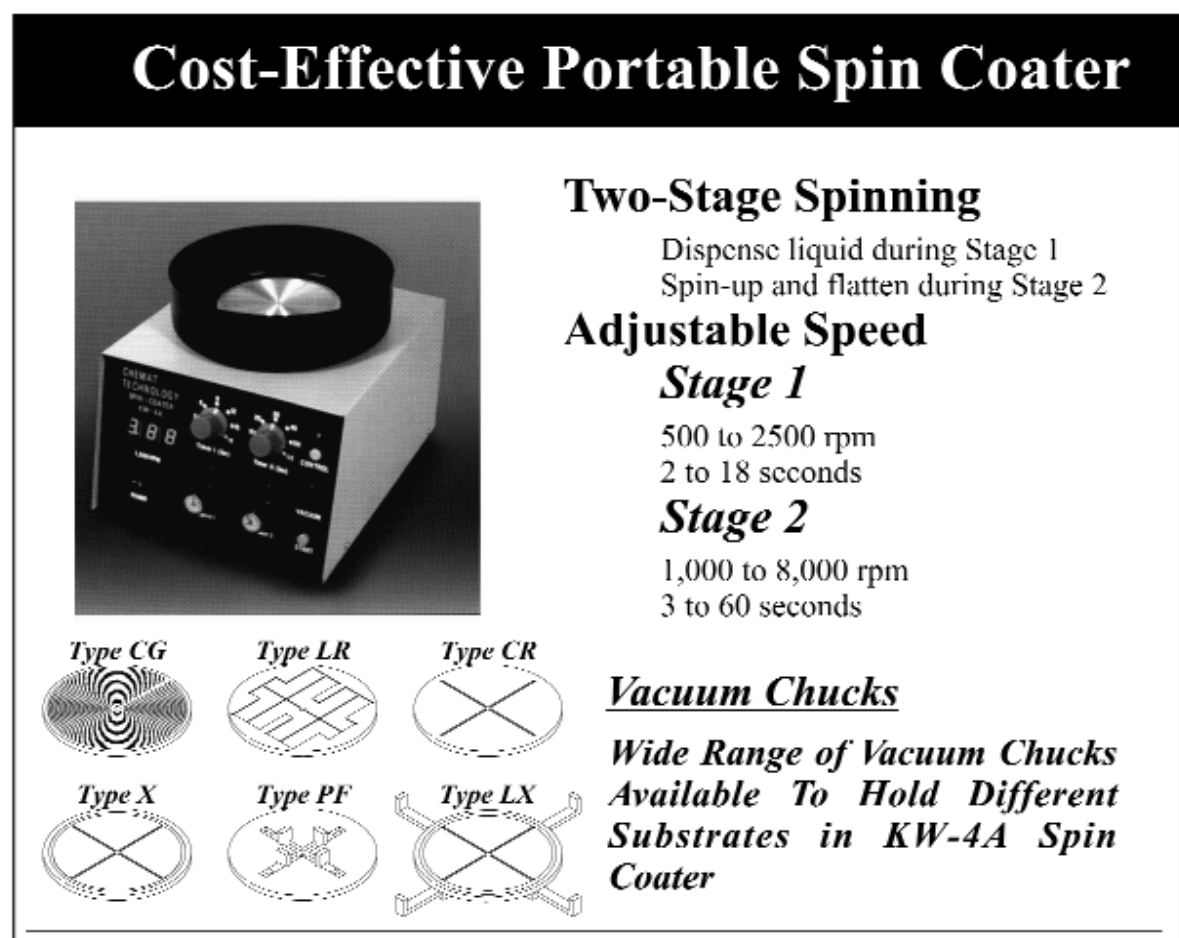

\title{
KW-4A SERIES PRODUCT LINE
}
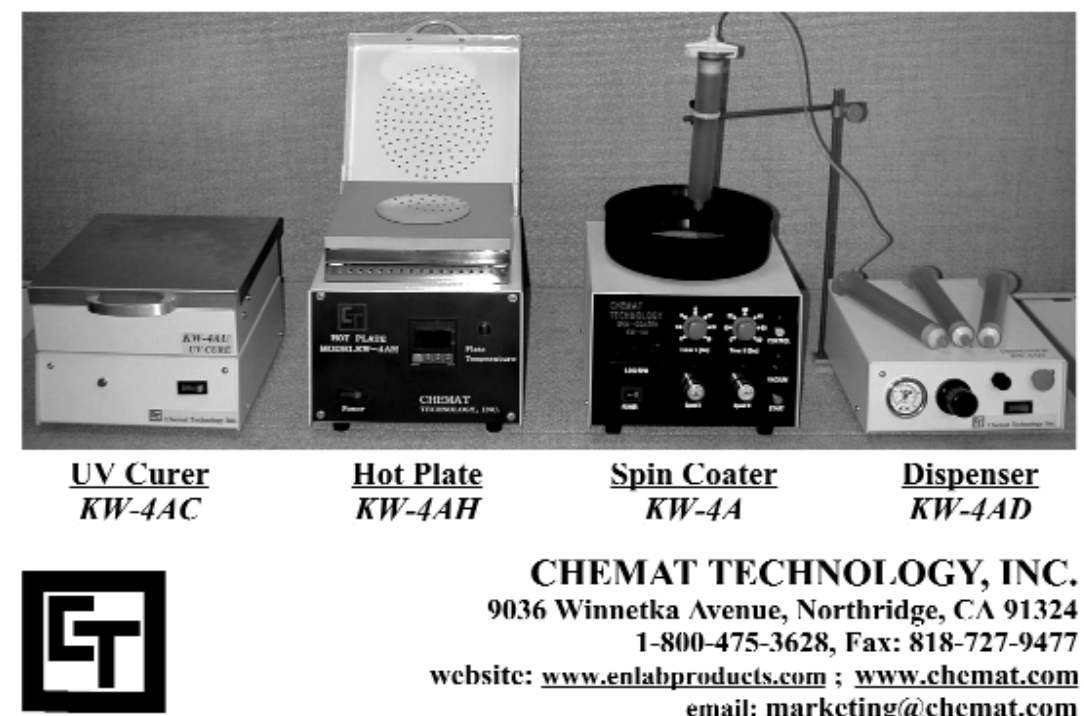

CHEMAT TECHNOLOGY, INC. 9036 Winnetka Avenue, Northridge, C $\Lambda$ 91324 1-800-475-3628, Fax: 818-727-9477 website: www.enlabproducts.com ; www.chemat.com email: marketing $(a)$ chemat.com

For more information, see http://www.mrs.org/bulletin_ads 